\title{
DÜBLIN
}

Technological University Dublin

ARROW@TU Dublin

2002-9

\section{An Environment for Mobile Context-based Hypermedia Retrieval}

James Carswell

Technological University Dublin, james.carswell@tudublin.ie

Follow this and additional works at: https://arrow.tudublin.ie/dmccon

Part of the Computer Sciences Commons

\section{Recommended Citation}

Carswell, J. (2002) An environment for mobile context-based hypermedia retrieval. 13th International Conference on Database and Expert Systems Applications (DEXA2002), IEEE CS Press, Aix en Provence, France,September 2-6.

This Conference Paper is brought to you for free and open access by the Digital Media Centre at ARROW@TU Dublin. It has been accepted for inclusion in Conference papers by an authorized administrator of ARROW@TU Dublin. For more information, please contact arrow.admin@tudublin.ie, aisling.coyne@tudublin.ie, gerard.connolly@tudublin.ie.

Funder: Enterprise Ireland through the Informatics

Programme 2001 on Digital Media

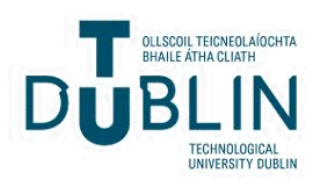


Dublin Institute of Technology

ARROW@DIT

Articles

Digital Media Centre

2002-09-01

\section{An environment for mobile context-based hypermedia retrieval}

James D. Carswell

Dublin Institute of Technology, jcarswell@dit.ie

\section{Recommended Citation}

Carswell, James D.:An environment for mobile context-based hypermedia retrieval. 13th International Conference on Database and Expert Systems Applications (DEXA2002); IEEE CS Press; Aix en Provence, France; September 2002

This Conference Paper is brought to you for free and open access by the Digital Media Centre at ARROW@DIT. It has been accepted for inclusion in Articles by an authorized administrator of ARROW@DIT. For more information, please contact yvonne.desmond@dit.ie, arrow.admin@dit.ie. 


\title{
An Environment for Mobile Context-Based Hypermedia Retrieval
}

\author{
James D. Carswell, Alan Eustace, Keith Gardiner, Eoin Kilfeather, Marco Neumann \\ Digital Media Centre, Dublin Institute of Technology, Ireland \\ jcarswell@dit.ie
}

\begin{abstract}
This paper proposes a novel solution to querying hyperlinked multimedia cultural heritage datasets based on the user's context. Context in this sense is defined as the user's location in virtual space and the particular mobile device being modeled together with user preferences or profile. The purpose is to automatically push relevant data from the database server to the client based on this comprehensive definition of the user's context. Consideration in regard to which mobile device is currently being modeled is a primary filter for determining what data will be sent and in what format. For example, image data will not be sent to a mobile phone and video will not be sent to a PDA. The CHI (Cultural Heritage Interfaces) project differs from many of the models encountered on the Web in that its primary focus is not the accurate $3 D$ rendering of $a$ street/landscape, but the simulation of such a physical reality to explore the adaptive hypermedia paradigm in the context of a spatial navigation interface.
\end{abstract}

\section{Introduction}

Currently there exists a variety of models on the Web that provide a virtual tour of a given landscape or attempt to provide an intuitive location-aware interface to a given dataset. [1,2,3,4,5,6] All of these efforts however rely on client-side Java applets to perform client-pull operations of static data from the database. In practice, this approach has shown to be unreliable as different implementations of the Java Virtual Machine often dictate the success of such a solution, and the data being sent to the client remains the same each time it is triggered by the user. A parallel can be drawn here with "static" websites, which contain fixed html content, i.e. each web page on the site remains the same on each visit. In contrast to this would be "dynamic" websites, which feed the user tailored information according to their needs or interests, generating the content of pages dynamically or "on-thefly".

To this end, the CHI (Cultural Heritage Interfaces) project draws on research in areas such as narrative intelligence [7], and artificial intelligence [8], as well as looking at computer game development [9], to see how these models can be applied to the arena of location/context-aware cultural heritage interfaces.

Indeed, the web/hypertext paradigm in the context of a spatial navigation interface is a useful one to consider for the $\mathrm{CHI}$ project, i.e. the fluidity of a dynamic website in terms of content, married to the narrative intelligence of a computer game, navigated spatially. The main purpose of this paper is to outline the system architecture and functional specifications of the CHI Demonstrator developed to perform this task.

Conceptually, the CHI Demonstrator will allow a user to navigate through a virtual 3D Dublin Street and view data relevant to his/her location in this space. The virtual street will contain the facades of buildings constructed and rendered using VRML (Virtual Reality Modeling Language). As the user moves within this 3D world, his location or virtual space coordinates are concurrently transformed into real world geographic coordinates (latitude/longitude). These lat/long coordinates are then passed as parameters for the construction of a query to the spatial database. The query will in effect retrieve all data from the database, within a certain tolerance, of the user's position in virtual 3D space, e.g. retrieve all data within $+/-10 \mathrm{~m}$ of these coordinates. The result of the query is returned to the user's hand held (mobile) device, which for the purpose of the CHI project, is a series of simulated hand held devices (e.g. cell phone, WAP phone, Pocket PC, PDA, etc.), displayed in parallel with the VRML 3D world on a standard desktop/laptop PC. (Figure 1)

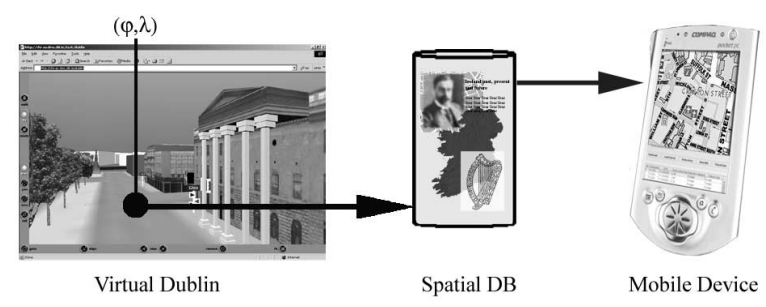

Figure 1 - Conceptual Overview of $\mathrm{CHI}$

The capabilities of the CHI Demonstrator are enhanced by adding the required functionality to allow for the spatial database itself to "drive" the user through the virtual streets of Dublin, based on some pre-selected 
theme, e.g. the "Easter Uprising of 1916". In this scenario, a query is constructed to retrieve all the data referring to this particular historical event and subsequently drives the user to the various locations within the virtual 3D world based on the spatial components of the relevant data. (Figure 2)

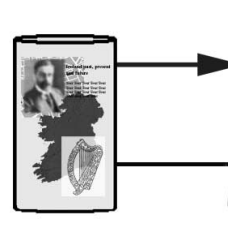

Spatial DB

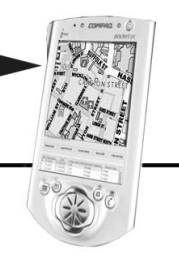

Mobile Device $(\varphi, \lambda)$

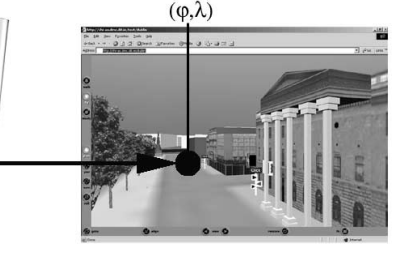

Virtual Dublin
Figure 2 - Enhanced CHI Demonstrator

A challenge in the presentation of complex and linked cultural heritage information exists in the tension between the random access to discrete data on the one hand with the desirability of developing narratively coherent linkage between data on the other. Although the event driven sequence of presented data do not themselves constitute a narrative, they do potentially form the building blocks for a narrative. In a classical narrative structure it is the agency of the "storyteller" that events are seen to have causality and ultimately to have coherence and meaning. [10] CHI is helping to develop some rules based "agentive" support for such coherent presentation. This will be discussed briefly later.

\section{Components of CHI}

To realise the CHI project technology demonstrator introduced above, the main components are implemented in a three-tier web-based architecture typical of spatially enabled enterprise applications, i.e. it comprises three layers, namely the Client Layer, the Application Server Layer, and the Database Layer. [11] (Figure 3)

All communications between the client layer and the database are conducted through the application server layer. The application, in our case the query building and query results formatting, is executed on the application server. The client communicates with the application server using the existing HTTP networking protocols.

Although a web server and a firewall have been incorporated in Figure 3, these should be considered as additional components that are not required in the base CHI system configuration and are only included to illustrate a complete web-based solution. For our purposes, the client, application server and database could in fact all physically reside on the same machine or indeed be split amongst multiple sites. Either configuration is entirely transparent to the user and both will allow for full access to the CHI demonstrator over the WWW.

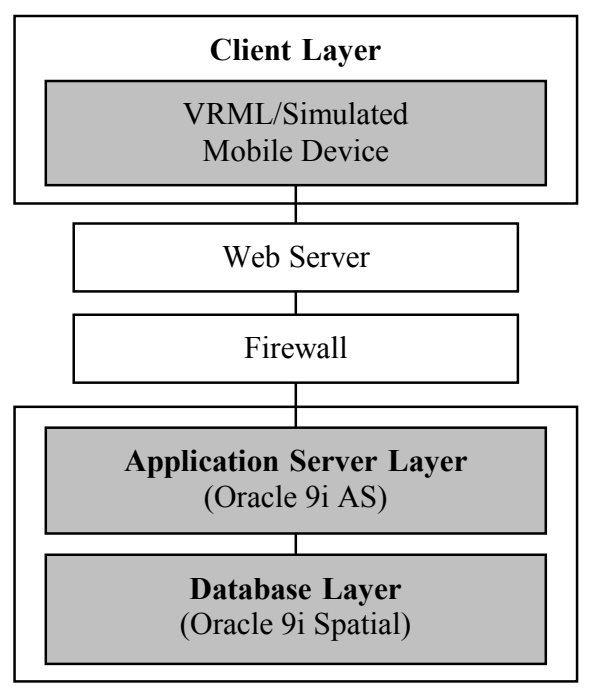

Figure 3 - Three-Tier Architecture of $\mathrm{CHI}$

\subsection{Client Layer}

The client layer will consist of a standard web browser to display the VRML model of Dublin streets. The various simulated mobile devices will also be displayed on the client, but except for the Web Browser simulation, will not be displayed within the same browser as the VRML model. Indeed the simulated hand-held devices may not be displayed within a browser at all and instead run as separate applications. Therefore, the VRML model could in fact be displayed on a separate monitor to that of the simulated device if desired.

Initially, the user's location in space and the particular mobile device being modeled will determine the user's context. The purpose therefore of this preliminary implementation of the demonstrator is to automatically push relevant data from the database layer to the client layer based on this limited contextual view. Consideration in regard to which mobile device is currently being modeled will determine what data will be sent and in what format. For example, image data will not be sent to a mobile phone and video will not be sent to a PDA. However, as one of the display devices to be modeled is a standard web browser, all types of multimedia information will be available for transmission in this case.

Choosing to simulate the mobile devices instead of actually implementing on the physical device allows for the accuracy and capabilities of these devices or context sensors to be emulated to any degree. For example, emulated location sensing devices can be more accurate than current technology allows. This flexibility allows application designers to use proposed or not yet ready sensors in the design of context-aware applications, a desirable component for location-aware services [13]. 
For the enhanced CHI Technology Demonstrator, the definition of the user's context is expanded to include user preferences or profile (e.g. the user's interests, age, task, etc.) in addition to the location and device display capabilities, bandwidth/protocol capabilities, etc. referred to above.

2.1.1. VRML. The Virtual Reality Modelling Language has been defined by the VRML Consortium as "an open standard for 3D multimedia and shared virtual worlds on the Internet."[12] It has been chosen as the implementation platform for the virtual representation component on the client side of the CHI project because it is a scene description language that describes the geometry and behavior of a 3D scene or "world" which in our case comprises the streets of Dublin. VRML "worlds" can range from simple objects to very complex scenes, but for transmission speed considerations, never include the entire geometry and all the features of the physical scene being modeled.

The location of the users viewpoint, while navigating within the VRML Dublin Street world, is used to simulate the users position in space. These virtual 3D coordinates are transformed into real-world latitude/longitude geographic coordinates using a 3rd order polynomial, which are in turn used to form the context-based query to the spatial database.

\subsection{Application Server Layer}

The application server implemented for the CHI project is Oracle 9iAS. This layer contains several Enterprise Java Beans (EJBs) illustrated in Figure 4 and described in the following.

The CHI session EJB is responsible for all communication with the client. As it is a stateful session EJB, an object of this type is instantiated for each user session. This EJB is responsible for monitoring changes in the user's context. For example, if the position of the user's location in virtual space changes, this EJB will transform the new coordinates into real-world (lat/long) space before passing it to the entity beans. Other relevant context data will also be passed at this time including the type of mobile device currently being simulated on the client and any user profiles that may exist.

SQL statements are needed to retrieve, from the spatial database, the information to be displayed on the client site. For example, when the user's context is received from the CHI EJB, a query is constructed and sent to the Oracle database engine for execution. The entity beans responsible for the construction, validation and execution of SQL statements are called SQL EJBs.

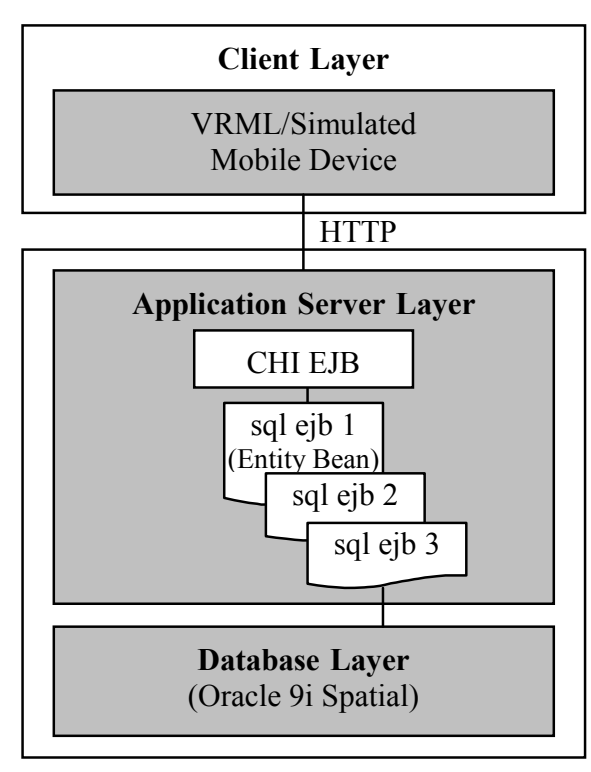

Figure 4 - Application Server Layer EJBs

A difficulty in creating a meaningful narrative hypermedia system is finding ways to mine information for different contexts. Portions of one piece of media may need to be presented separately according to user context. For example, a user's context may include their interest in architecture, but not in politics. In this scenario, the SQL EJB builds a query in connection with the architectural information about a building, but its political relevance is ignored.

The query results are returned to the CHI EJB via the SQL EJB which organizes the results into (simulated) device specific format. If the same query is run again (i.e. no change in user context), the CHI EJB re-invokes the SQL EJB that was previously instantiated. The parsed version of the previously executed SQL command is cached by the SQL EJB thereby eliminating the need to re-interpret the query. Bind variables are used to optimise the execution of the SQL query and to assign values to object variables.

The SQL EJB also formats the query results for a selection of platforms as already described, including WAP, PDA, and a standard web browser. XML is used in conjunction with XSLT (extensible stylesheet language transformations) to convert XML data into the various required formats e.g. HTML, WML, etc. The advantage of using XSLT for such a scenario is that the underlying XML data structure remains the same, and by applying XSL stylesheets the data is formatted on the fly to whatever format is desired by using an XSLT processor.

There are a number of XSLT processors available, and Sun Microsystems' Java API for XML Processing (JAXP) provides a standard Java interface to many of them. [13] 
Figure 5 illustrates the process of transforming XML into a desired format.

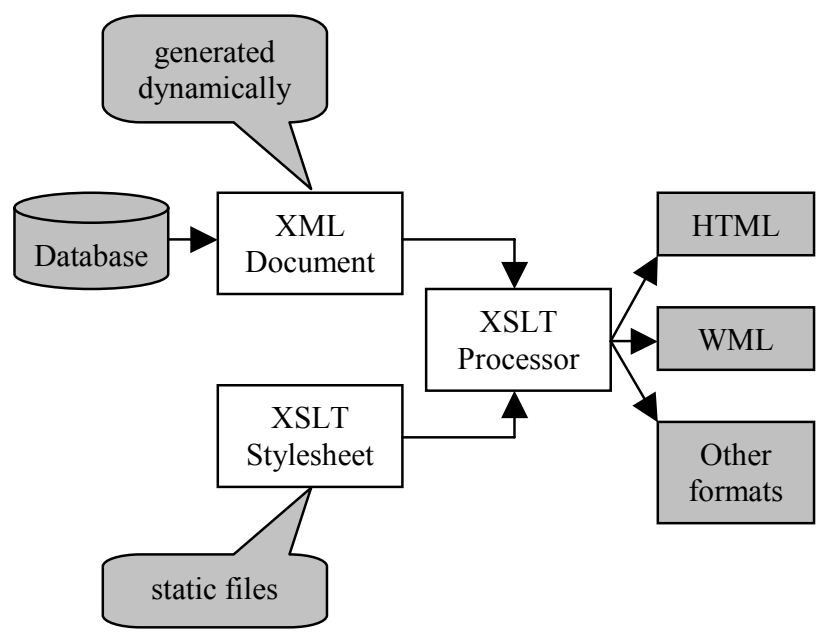

Figure 5 - XML Formatting Workflow

\subsection{Database Layer}

The database implemented for the CHI project is Oracle $9 \mathrm{i}$ which provides spatial object type storage, SQL access, spatial operations, and indexing as well as map projections and coordinate systems support. [14] Through this functionality, spatial queries are efficiently executed without the additional overhead of maintaining coordinate information separate from the attribute data. This is accomplished by defining the attribute information (hypermedia) as a spatial data type (which implies associated coordinate data), e.g. either a point, line, or area. The primary advantage of spatial data types is that queries can be restricted to a pre-defined geographical area, e.g. within a $10 \mathrm{~m}$ radius of a given location. By exploiting the spatial indexing mechanisms inherent to Oracle 9i, which essentially organises the information within the database tables according to their geographic location, all location relevant data can be retrieved efficiently.

The hypermedia objects stored in the CHI project database (together with their spatial component) comprise an "historic walking tour of Dublin". [15] Such a tour can begin and end at specific times and places and pass specific landmarks along the way. As each of the landmarks is encountered in turn, a particular "story object" will be retrieved about its historical significance. It is the text of this "story" that will comprise the bulk of the data stored in the $\mathrm{CHI}$ database layer. A challenging aspect to this research is the investigation of the methodologies for retrieving these story objects both automatically and coherently as their positions in space are approached. To accomplish this task successfully, the causality of the localised series of events is considered.

\section{Narrative in Hypermedia}

In classical narration and in traditional media like film and theatre the causal and temporal presentation of story objects is wholly prescribed by the author. The user passively receives, determined by the camera position and succession of events, the narrative of the film or play. The development of hypertextual technologies and structures has allowed the user (or 'reader' in textual analysis terms) to disrupt this narrative assumption. The involvement of the user in the production of the story "text" is not unique to hypertextual applications and has been studied in other guises as inter-textuality and reader response. In considering the role of the user however we must now take into account the possibility of the user's context now determining the presentation of story objects. Using narrative studies as a basis we aim to consider the role and context of the user in the understanding of cultural narratives within the $\mathrm{CHI}$ project [16] [17]

The use of narrative within hypertextual constructs must consider too seemingly conflicting strategies. Firstly there is the need to maintain coherence through canonic narrative cues [18]. These cues such as temporality (the presentation of narrative elements in chronological sequence), causality or narrative logic (events or sequences are linked semantically on some level) and space (events and stories take place in a real space which must normally be depicted). The maintenance of temporal and causal continuity is normally the responsibility of the author who arranges story objects in a predetermined manner. The success or failure of the narrative coherence is determined by the reader/user's interpretation of those elements. The conflicting strategy in a hypertextual construct (such as the CHI demonstrator) is the development of a freer and more associative relationship between elements. This hypertextuality on the one hand weakens the narrative function while on the other strengthens the associative possibilities within the hypertext.

The principal reason to develop a broader narrative strategy using context awareness is that for some cultural texts the association between the story elements and space is strong. This is especially true of the text of the 1916 Easter Rising. In this historical text the events of the rebellion take place in a relatively small area in the center of Dublin. The story objects within the database can be therefore given metadata that associates the story object both semantically and spatially. For example by associating an object with the theme "social struggle", or "nationalism" but also associating it with place (using a coordinate system). In contrast to most hypertextual 
systems the spatial continuity of the narrative is preserved while the user moves through space. While this on its own provides only a crude form of coherence it is nevertheless possible to build on this to allow further narrative possibilities.

Inherent in many agentive narrative coherency strategies is the concept of 'emergence' [19]. Emergence indicates the possibility of a coherence developing from the associated flow of story elements within a structured yet interactive context. The use of the location context of a user is just such a coherency strategy. However the spatial continuity alone of such a presentation is unlikely to prove satisfactory to the user. In the $\mathrm{CHI}$ demonstrator an aim is to determine how theses coherence 'rules' can be combined to present satisfactory narrative experiences to the user. Rules governing spatial nearness (to a story object), semantic nearness (of story objects to each other) and tour history (using state rules to prevent story objects from being replayed) are combined to experiment heuristically with the 'in-process' narrative. An interesting avenue of investigation is in the review of the user's visits to locations which can be stored by the application. This allows a feedback mechanism that can subsequently be used to add new layers of information.

\section{Conclusion}

In this paper, we have presented a novel solution for accessing and querying multimedia cultural heritage datasets. The $\mathrm{CHI}$ project seeks to demonstrate ways to represent cultural heritage information in mobile hypermedia environments in a coherent manner. In order to fulfill this requirement, context-based retrieval and narrative structuring features are deployed in a three-tier web-based architecture.

The motivation for the project is evident; the advances in the information and telecommunications technology serve users ever more with globally accessible and timely data. But the extraction of meaningful information from this accumulated data requires new strategies to be developed. The reduction of complexity, in system architecture, data structure and usability, is the key element in designing new forms of human computer interaction and knowledge representation. Consequently the introduction of a mobile, context-based application such as CHI, capable of location determination and user reference, offers such a strategy: it allows for the forming and recombining of relevant data fragments into a coherent information flow.

Acknowledgements: Support for this research from Enterprise Ireland through the Informatics Programme 2001 on Digital Media is gratefully acknowledged.

\section{References}

[1] The Glasgow Directory. 2001 http://iris.abacus.strath.ac.uk/glasgow/

[2] Methana 3D. Touristic information system. 2001. http://www.karto.ethz.ch/ hm/methana/version05/methana. html.

[3] VIS - Vienna City 3D . 2001. http://www.karto.ethz.ch/neumann/cartography/vis/wien st art first.html

[4] e-Berlin. City Information Systems. 2001. http://www.e-berlin.de

[5] Wetzlar. 2001, http://www.wetzlarvituell.de/

[6] Virtual City of Tampere. 2001. http://www.uta.fi/hyper/projektit/tred/english/index.htm

[7] Dautenhahn, K., Coles, S. J., "Narrative Intelligence from the Bottom Up: A Computational Framework for the Study of Story-Telling in Autonomous Agents", Journal of Artificial Societies and Social Simulation, vol. 4, no. 1. 2001.

http://www.soc.surrey.ac.uk/jasss/4/1/1.html

[8] Treister-Goren, A., Hutchens, J. L., "Creating AI: A unique interplay between the development of learning algorithms and their education", http://www.a-i.com/data/59Ai_Research_04.pdf

[9] Smith, H., "The Future of Game Design: Moving Beyond Deus Ex and Other Dated Paradigms" http:/www.igda.org/Endeavors/Articles/hsmith intro.htm

[10] Kilfeather, E., "Hypertext, Narrative and Coherence: The role of the reader and writer in the practice of hypertext", M. Phil Thesis. DIT. 1998. http://www.dmc.dit.ie/eoin/interview/Hypertext and Text6 $\underline{1 . p d f}$

[11] Bertolotto, M., Carswell, J. D., McGeown, L., McMahon, J., "iSmart+iSIS: Deploying Integrated Web-Based Spatial Applications Within an Oracle Database Environment", International Workshop on Web Geographical Information Systems (WGIS2001), IEEE CS Press, Kyoto, Japan. 2001.

[12] VRML Consortium: http://www.vrml.org/

[13] Sun Microsystems' Java API for XML Processing (JAXP): http://java.sun.com/xml/download.html

[14] Rigaux, P., Scholl, M., Voisard, A., "Spatial Databases": 2002: Academic Press, Morgan Kaufmann Publishers. pp. 352-360

[15] Kostick, C., Collins, L., "The Easter Uprising: A Guide to Dublin in 1916", The O'Brien Press, Dublin. 2000.

[16] Ryan, ML., "Beyond Myth and Metaphor -The Case of Narrative in Digital Media", Game Studies, Issue 1. July 2001. http://www.gamestudies.org/0101/ryan/

[17] Kolstrup, S., "Interactive Narratives", Qvortrup, Lars (Ed): Virtual Interaction. Interaction in Virtual Inhabited $3 D$ Worlds. 2001.

[18] Bordwell, D., "Narration in the Fiction Film", Routledge. London. 1985

[19] Murtagh, M., "The Automatist Storytelling System: Putting the Editor's Knowledge in Software", MIT Media Lab, M.Sc. Thesis, 1996 